\title{
Zur kolorimetrischen Albuminbestimmung im Serum
}

\author{
Von \\ Z. F. Ch. Kachani \\ Aus dem Hygiene-Institut der Universität Kiel (Direktor: Prof. Dr. H. Gärtner)
}

(Der Schriftleitung zugegangen am 4. September 1963)

\begin{abstract}
Es wird über eine einfache und schnell durchführbare Methode berichtet, bei der sich mittels der kolorimetrischen Bestimmung quantitative, reproduzierbare Werte für das Albumin im Serum ergeben. Die gemessene Fraktion besteht, wie sich immunelektrophoretisch zeigen läßt, aus reinem Albumin. Vergleichsuntersuchungen bei 60 normalen und pathologischen Seren zeigten eine gute Ubereinstimmung des neuen Verfahrens mit den Ergebnissen der Elektrophorese.
\end{abstract}

A simple, rapid method is reported for the quantitative, colorimetric determination of serum albumin. The values are reproducible. The measured fraction consists of pure albumin, as shown by immunoelectrophoresis. Comparison of results for 60 normal and pathological sera showed good agreement between the new method and electrophoresis.

Zur quantitativen Fraktionierung der Serumeiweißkörper hat das Fällungsverfahren mit organischen Lösungsmitteln wegen der uneinheitlichen Fraktionen im klinisch-chemischen Laboratorium kaum Eingang gefunden, und auch die Trennung mittels der Ultrazentrifuge ist aus praktischen Gründen für die Routinereihenuntersuchung nicht durchführbar. Durchgesetzt haben sich in der Klinik am meisten die Aussalzmetboden und die Elektrophorese. - Die Aussalzverfahren sind zeitraubend. Auch entstehen dabei keine elektrophoretisch reinen Albuminfraktionen, wie wir bei unseren Vergleichsuntersuchungen in Übereinstimmung mit PILLEMER und Mitarbeitern (1) feststellen konnten. Bei der Verwendung von Natriumsulfat als Fällungsmittel war außerdem das Verhältnis Albumin zu Globuline nicht demjenigen der Elektrophorese vergleichbar. So hat sich die Elektrophorese trotz des apparativen Aufwandes in den vergangenen $15 \mathrm{Jahren}$ weit verbreitet.

Es schien uns deshalb wichtig, eine Methode zu entwickeln, die es mit wenig Zeit- und Kostenaufwand und ohne spezielle Apparaturen ermöglicht, Serumeiweißkörper quantitativ zu fraktionieren, so da $\beta$ die Ergebnisse mit denjenigen der Elektrophorese vergleichbar sind. - Hierbei gingen wir von einer Mitteilung von Lavine (2) sowie Delaville und Mitarbeitern (3) aus, die zuerst beobachten konnten, daß das mit Trichloressigsäure („TES“) präzipitierte Rinder- bzw. Serumalbumin durch Zusatz von polaren Lösungsmitteln löslich wird. Die Feststellung, daß durch TES gefällte Albumine bei Zugabe von Aceton und Alkohol wieder löslich werden, ist von Schwert (4), KORNER und Mitarbeiter (5) zur Herstellung von biologisch einwandfreien, hochgereinigten Albuminen benutzt, und von verschiedenen Seiten $(6,7)$ als Methode der Wahl zur Albumingewinnung mit großer Ausbeute empfohlen worden, weil durch die TES-ÄthanolBehandlung das Albumin seine physikalisch-chemischen Eigenschaften nicht verändert. Elektrophoretische Be- weglichkeit, Farbstoffbindungsvermögen, Sedimentationskonstante, Löslichkeit, Kristallisierbarkeit und immunologische Eigenschaften bleiben nachweislich dieselben, wie diejenigen der unbehandelten Albumine $(5,8,9)$.

Es war zu prüfen, ob eine kolorimetrische Albuminbestimmung nach TES-Äthanol-Behandlung möglich ist und diese quantitative, reproduzierbare Werte erbringt, Dabei mußten wir zunächst feststellen, daß das von SCHWERT (4) und anderen Autoren benutzte Extraktionsverfahren sich für die kolorimetrischen Bestimmungen wegen der Trübung, die nach Zusatz des Biuretreagenzes entstand, nicht eignet. Nach weiteren Versuchen gelang es uns schließlich, folgende Methodik zu entwickeln.

\section{Methodik}

$\mathrm{Zu} \quad 4,8 \mathrm{ml}$ TES- $\AA$ thanol-Lösung $(0,4 \mathrm{~g}$ Trichloressigsäure in $100 \mathrm{~m} l$ 97-proz. Athanol p. a.) $0,2 \mathrm{~m} l$ Serum ganz langsam und tropfenweise direkt oberbalb des Flüssigkeitsspiegels zugeben. Nicht schütteln! Nach 5 Min. das Präzipitat mit einem Glasstab gut in der Flüssigkeit verteilen. 5 Min. bei 5000 U/Min. zentrifugieren, Uberstand dekantieren. In einem Reagenzglas $5 \mathrm{~m} l$ Biuretreagenz und $2,5 \mathrm{~m} l \mathrm{NaCl}-\mathrm{NaOH}-L o ̈ s u n g(0,1 \mathrm{~g} \mathrm{NaOH}$ in $100 \mathrm{~m} l$ 1,8-proz. $\mathrm{NaCl}$-Lösung) vorlegen. Erst jetzt mit $2,5 \mathrm{ml}$ von dem klaren Uberstand ganz langsam überschichten. Durch sehr schnelles Umkippen des Röhrchens (wichtig!) den Inhalt gut mischen. Die Flüssigkeit muß nach Entweichen der Gasbläschen völlig klar sein. Nach Ablauf von 30 Min. gegen den Leerwert, der aus $5 \mathrm{~m} l$ Biuretreagenz und $5 \mathrm{ml} \mathrm{NaCl}$-Lösung besteht, ablesen, und zwar in derselben Art, wie es bei der Gesamteiweißbestimmung nach Weichselbaum üblich ist (10). (Photometer Eppendorf, $20 \mathrm{~mm}$ Küvetten, Filter $546 \mathrm{~m} \mu$.)

Um festzustellen, ob zwischen dem auf diese Weise ermittelten Albumingehalt des Serums und der Farbintensität ein gesetzmäßiger Zusammenhang besteht, wurden 11 Eichlösungen zwischen 0,5 bis $5,0 \mathrm{~g} \%$ mit humanem Serumalbumin ${ }^{1}$ ) in physiol. Kochsalzlösung hergestellt. Zur Kontrolle, ob die im Serum vorhandenen

1) Behringwerke. 
Globuline das Ergebnis beeinflussen, wurde den Eichlösungen in einer Parallelserie außerdem normales Serum zugesetzt und hier nach Ablauf der Reaktionszeit gegen dasselbe Serum mit entsprechender Verdünnung, aber ohne Albuminzusatz, als Leerwert abgelesen. Der gradlinige Verlauf der Eichkurve, der sich aus beiden Serien ergab, zeigte, daß das Lambert-Beersche Gesetz $z$ wischen 0,5 und $5,0 \mathrm{~g} \%$ Albumine erfüllt ist; dieses bedeutet eine direkte Gesetzmäßigkeit zwischen der Extinktion und dem Albumingehalt des Serums. Auf Grund der Eichkurve wurde der Faktor 1,222) ausgerechnet, d. h.: Extinktion $\times$ Faktor des Biuret nach Weichselbaum ${ }^{3}$ ) $\times 1,22=\mathrm{g} \%$ Albumin.

Um nachzuprüfen, ob die erzielten Eiweißwerte durch eine reine und einheitliche Albuminfraktion bedingt waren, wurden nach der Pervaporation und Dialyse mit den extrahierten Albuminen eine Immunelektrophorese und eine Papierelektrophorese angesetzt. Die Abbildungen 1 und 2 zeigen, daß in beiden Verfahren das extrahierte Eiweiß sich als eine reine Albumin-Fraktion darstellte.

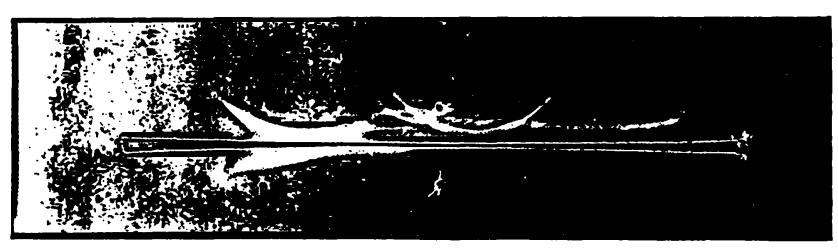

Abb. 1

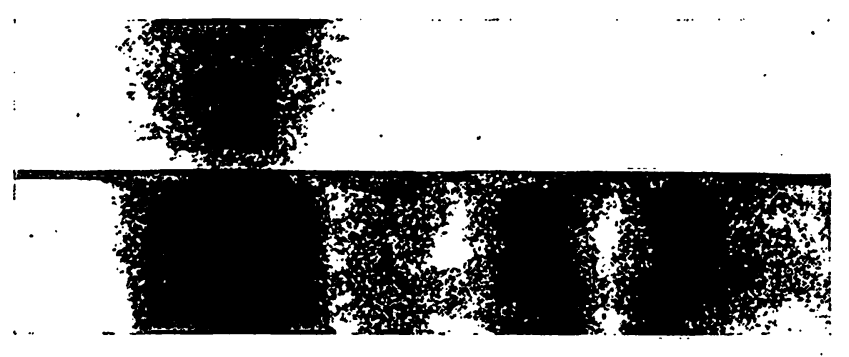

Abb. 2

Schließlich wurde durch Vergleichsuntersuchungen bei 60 normalen und pathologischen Seren geprüft, ob die Ergebnisse des TES-Äthanol-Verfahrens denjenigen der Elektrophorese ebenbürtig sind. Jede der beiden Methoden wurde in doppeltem Ansatz durchgeführt; die Mittelwerte wurden miteinander verglichen. Wie die Tabelle 1 zeigt, besteht eine gute Übereinstimmung zwischen den Ergebnissen der beiden Verfahren. Die Schwankungen liegen innerhalb der Fehlerbreite der beiden Methoden. Die Fehlerbreite von $\pm 0,05 \mathrm{~g} \%$ für das TES-Äthanol-Verfahren war weit geringer als diejenige der Elektrophorese. - Bẹi pathologischen Seren mit stark verminderten Albuminwerten fielen die Ergebnisse der TES-Äthanol-Methode durchschnittlich

2) Dieser Faktor entspricht einer Ausbeute von $82 \%$.

3) Dieser Faktor muß für jedes neue Biuretreagenz neu bestimmt werden, deshalb haben wir auf Angabe einer konstanten Zahl verzichtet.
Tab. 1

Gefundene Albumingehalte

Lfd. Gesamt- TES-Äthanol-Methode Elektrophorese

Nr. Eiweiß

\begin{tabular}{lllll}
$\mathrm{g} \%$ & $\mathrm{~g} \%$ & rel. $\%$ & $\mathrm{~g} \%$ & rel. $\%$ \\
\hline
\end{tabular}

\begin{tabular}{|c|c|c|c|c|c|}
\hline 1 & 6,293 & 3,62 & $5 \hat{7}$ & 3,52 & 56 \\
\hline 2 & 6,15 & 3,7 & 60 & 3,69 & 60 \\
\hline 3 & 6,15 & 3,2 & 52 & 3,26 & 53 \\
\hline 4 & 5,996 & 3,5 & 58 & 3,54 & 59 \\
\hline 5 & 6,10 & 3,55 & 58 & 3,54 & 58 \\
\hline 6 & 5,637 & 3,45 & .61 & 3,44 & 61 \\
\hline 7 & 5,945 & 3,375 & 56 & 3,21 & 54 \\
\hline 8 & 6,211 & 3,5 & 57 & 3,66 & 59 \\
\hline 9 & 6,15 & 3,55 & 59 & 3,63 & 59 \\
\hline 10 & 6,45 & 3,62 & 56 & 3,74 & 58 \\
\hline 11 & 5,84 & 3,75 & 64 & 3,74 & 64 \\
\hline 12 & 6,04 & 3,62 & 60 & 3,62 & 60 \\
\hline 13 & 6,15 & 3,125 & 53 & 3,44 & 56 \\
\hline 14 & 6,047 & 3,8 & 63 & 3,81 & 63 \\
\hline 15 & 6,76 & 3,75 & 56 & 3,92 & 58 \\
\hline 16 & 6,04 & 3,35 & 56 & 3,44 & 57 \\
\hline 17 & 6,15 & 4,125 & 67 & 4,12 & 67 \\
\hline 18 & 6,04 & 3,75 & 62 & 3,68 & 61 \\
\hline 19 & 6,662 & 4,375 & 65 & 4,40 & 66 \\
\hline 20 & 3,28 & 2,125 & 64 & 2,07 & 63 \\
\hline 21 & 6,498 & 4,125 & 63 & 4,16 & 64 \\
\hline 22 & 6,46 & 4,07 & 63 & 4,01 & 62 \\
\hline 23 & 5,945 & 3,67 & 60 & 3,39 & 57 \\
\hline 24 & 5,47 & 3,62 & 66 & 3,56 & 65 \\
\hline 25 & 6,25 & 3,125 & 50 & 3,25 & 52 \\
\hline 26 & 6,56 & 4,125 & 62 & 4,07 & 62 \\
\hline 27 & 6,355 & 4,525 & 71 & 4,45 & 70 \\
\hline 28 & 5,424 & 3,425 & 63 & 3,42 & 63 \\
\hline 29 & 6,622 & 3,425 & 52 & 3,51 & 53 \\
\hline 30 & 6,7 & 3,5 & 52 & 3,69 & 55 \\
\hline 31 & 5,53 & 3,625 & 65 & 3,65 & 66 \\
\hline 32 & 6,15 & 3,43 & 55 & 3,38 & 55 \\
\hline 33 & 5,54 & 3,25 & 58 & 3,05 & 55 \\
\hline 34 & 6,4 & 4,175 & 65 & 4,10 & 64 \\
\hline 35 & 6,6 & 3,875 & 58 & 3,83 & 58 \\
\hline-36 & 6,7 & 3,8 & 56 & 3,75 & 56 \\
\hline 37 & 6,15 & 4,0 & 65 & 4,00 & 65 \\
\hline 38 & 5,59 & 3,25 & 58 & 3,53 & 56 \\
\hline 39 & 6,4 & 3,5 & 54 & 3,58 & 56 \\
\hline 40 & 5,2 & 2,7 & 52 & 2,7 & 52 \\
\hline 41 & 6,8 & 4,7 & 69 & 4,7 & 69 \\
\hline 42 & 7,1 & 4,7 & 66 & 4,6 & 65 \\
\hline 43 & 7,0 & 3,3 & 47 & 3,3 & 48 \\
\hline 44 & 6,2 & 4,25 & 68 & 4,2 & 68 \\
\hline 45 & 6,0 & 3,5 & 58 & 3,48 & 58 \\
\hline 46 & 7,1 & 3,12 & 44 & 3,2 & 46 \\
\hline 47 & 6,66 & 3,87 & 58 & 3,89 & 58 \\
\hline 48 & 6,86 & 3,9 & 57 & 3,85 & 56 \\
\hline 49 & 6,1 & 2,37 & 39 & 2,6 & 43 \\
\hline 50 & 8,14 & 2,58 & 32 & 2,68 & 33 \\
\hline 51 & 7,76 & 4,06 & 52 & 4,0 & 52 \\
\hline 52 & 7,29 & 4,18 & 57 & 4,11 & 56 \\
\hline 53 & 5,74 & 3,62 & 63 & 3,2 & 55 \\
\hline 54 & 7,79 & 4,61 & 59 & 4,63 & 59 \\
\hline 55 & 6,86 & 4,42 & 64 & 4,32 & 62 \\
\hline 56 & 5,64 & 2,55 & 45 & 2,54 & 45 \\
\hline 57 & 7,65 & 2,05 & 27 & 2,29 & 30 \\
\hline 58 & 6,9 & 2,625 & 38 & 2,69 & 39 \\
\hline 59 & 7,318 & 2,750 & 37 & 2,71 & 37 \\
\hline 60 & 6,662 & 3,85 & 58 & 4,0 & 60 \\
\hline
\end{tabular}

Z. klin. Chem. / 2. Jahrg. 1964 / Heft 3 
etwas niedriger aus als die der Elektrophorese. Da für die Elektrophorese das Beersche Gesetz bei der Transparenzphotometrie nicht streng erfüllt ist $(11,12,6$, $16,13,8)$, ist diese Differenz erklärlich. - Hämolytische Seren, auch solche mit leichten Hämolysegraden, sind für die TES-Äthanol-Methode wie für die Elektro- phorese $(14,15)$ ungeeignet. So ist man mit dem TESÄthanol-Verfahren in der Lage, schnell und exakt den Albumingehalt des Serums festzustellen und durch Subtrahieren dieses Wertes vom Gesamteiweiß des Serums den Globulinanteil und damit das Verhältnis Albumin zu Globulin zu ermitteln.

\title{
Literatur
}

1. Pillemer, L. und M. C. Hutchinson, J. biol. Chemistry 158 , 299 (1945). - 2. Lavine, S., Arch. Biochem. Biophysics 50, 515 (1954). - 3. Delaville, M., G. Delaville und J. Delavirle, Année pharmaceut. Paris, 12, 109 (1954). - 4. Schwert, G. W., J. Amer. chem. Soc. 79, 139 (1957). - 5. KorNER, A. und J. R. Debro, Nature (London) 178, 1067 (1956). - 6. Owen, J. A., Analyst, 81, 26 (1956). - 7. MrChAEL, S. E., IV. Intern. Biochem. Kongreß, Wien (1958). - 8. WALSCH, J. R., F. L. Humoller und A. L. DunN, J. Laborat. Clin. Med., S. Louis 46, 772 (1955). 9. Kallee, E., F. Lohss und W. OppermanN, Z. Naturforsch.
Teil B, 12, 777 (1957). - 10. Weichselbaum, T. E., Amer. J. Clin. Path. (Techn. Sect.) 10, 40 (1946). - 11. Fuchs, W. und A. FlACH, Klin. Wschr. 33, 903 (1955). - 12. Osterhuits, H. K., J. Laborat. Clin. Med., S. Louis 44, 280 (1956). - 13. Schulz, D. M. und M. Holdcraft, Amer. J. Clin. Path. 26, 215 (1956). - 14. SANDKüHLER, S., Dtsch. med. J. 13, 266 (1962). - 15. SCHOEN, R. und $\mathrm{H}$. SüDHOF, Biochemische Befunde in der Differentialdiagnose innerer Krankheiten. Georg Thieme Verlag, Stuttgart (1962). 16. Hinsberg, K. und K. Lang, Medizinische Chemie. 3. Auflage S. 996, Urban \& Schwarzenberg, München, Berlin (19.57).

Dr. med. Z. F. Ch. Kachani $23 \mathrm{Kiel}$

Brunswiker Str. 2-6

\section{Sauerstoff-Aufnahme von Humanplasma nach Zugabe von Kobalt}

\author{
Von \\ J. Dittmann und P. Sachtreben \\ Aus der Universitäts-Kinderklinik Homburg, Saar, und der Landesklinik Neunkirchen-Koblhof, Saar \\ (Direktor: Prof. J. B. Mayer)
}

(Der Schriftleitung zugegangen am 7. Februar 1964)

\begin{abstract}
Gibt man $6 \mu \mathrm{Mol} \mathrm{CoSO}{ }_{4}$ gelöst in $0,2 \mathrm{~m} l$ Wasser unter $\mathrm{O}_{2}$ bei $38^{\circ} \mathrm{zu} 0,6 \mathrm{~m} l$ Plasma, so wird die $\mathrm{O}_{2}$-Aufnahme des Plasmas stark stimuliert. Gibt man die gleiche Menge CoSO ${ }_{4}$ zu der entsprechenden Menge, nämlich $1 \mathrm{ml}$, Blut, so bleibt der beschriebene Effekt aus.

The addition of $6 \mu \mathrm{mol}$. of $\mathrm{CoSO}_{4}$ in $0.2 \mathrm{ml}$. of water to $0.6 \mathrm{~m} /$. of plasma under $\mathrm{O}_{2}$ at $38^{\circ}$ causes a marked stimulation of the $\mathrm{O}_{2}$-uptake of the plasma. If the same amount is added to a corresponding amount of blood $(1 \mathrm{ml}$.), there is no effect.
\end{abstract}

Wir haben die Sauerstoff-Aufnahme von Blutzellen in Gegenwart verschiedener Kobalt-II-Ionen-Konzentrationen manometrisch gemessen und dabei folgende Beobachtung gemacht: Gibt man $6 \mu \mathrm{Mol} \mathrm{Co}^{2+}$ gelöst in $0,2 \mathrm{~m} l$ Wasser zu $1 \mathrm{~m} l$ Blut, so wird die folgende $\mathrm{O}_{2}-$ Aufnahme gegenüber den Kontrollen ohne $\mathrm{Co}^{2+}$-Zusatz etwas erhöht; gibt man die Lösung von $6 \mu \mathrm{Mol} \mathrm{Co}^{2+}$ in $0,2 \mathrm{~m} l$ Wasser jedoch zu der entsprechenden Menge, nämlich $0,6 \mathrm{~m} l$ Plasma, so setzt nach einer gewissen Inkubationszeit eine sehr kräftige $\mathrm{O}_{2}$-Aufnahme ein.

\section{Methode}

Blut wurde aus der Armvene in eine mit Heparin benetzte Spritze gezogen und anschließend sofort in vorbereitete Kegelgefäße von etwa $15 \mathrm{~m} l$ Inhalt pipettiert. Für die Versuche mit Plasma wurde das Blut unmittelbar nach der Entnahme $10 \mathrm{Min}$. lang bei etwa
$3000 \mathrm{~g}$ zentrifugiert. Genau $30 \mathrm{Min}$. nach Blutentnahme wurden die Gefäße in den Thermostaten gehängt und anschließend $5 \mathrm{Min}$. ohne Schütteln bei $38^{\circ}$ mit reinem $\mathrm{O}_{2}$ durchströmt. $50 \mathrm{Min}$. nach Entnahme wurde die Schüttelung der Gefäße begonnen: Warburg. Apparat Typ S 85 der Fa. Braun, 100 Schüttelungen pro Min., Amplitude $2 \mathrm{~cm}$. Genau $60 \mathrm{Min}$. nach Blutentnahme erfolgte die erste Manometer-Ablesung, genau $90 \mathrm{Min}$. nach Blutentnahme wurde aus dem Seitenanhang $\mathrm{Co}^{2+}$ zugegeben. In der graphischen Darstellung gilt der Augenblick des Zukippens als Versuchsbeginn.

Inbalt der Gefäße: Trog aller Gefäße $0,1 \mathrm{ml} 1 n \mathrm{KOH}$. Tabellenwerte in $\mathrm{ml}$.

\begin{tabular}{lcccc}
\hline & $\mathrm{I}$ & $\mathrm{II}$ & $\mathrm{III}$ & IV \\
\hline Hauptraum: & $1 \mathrm{Blut}$ & $1 \mathrm{Blut}$ & $0,6 \mathrm{Plasma}$ & $0,6 \mathrm{Plasma}$ \\
Anhang: & $0,2 \mathrm{H}_{2} \mathrm{O}$ & $0,2 \mathrm{H}_{2} \mathrm{O}$ & $0,2 \mathrm{H}_{2} \mathrm{O}$ & $0,2 \mathrm{H}_{2} \mathrm{O}$ \\
& & mit $6 \mu \mathrm{Mol}$ & & mit $6 \mu \mathrm{Mol}$ \\
& & $\mathrm{CoSO}_{4}$ & & $\mathrm{CoSO}_{4}$ \\
\hline
\end{tabular}

